\title{
The need and availability of support systems for physicians involved in a serious adverse event
}

\author{
Esther H.M. Leferink ${ }^{* 1,2}$, Aline Bos ${ }^{2}$, Martijn P. Heringa ${ }^{3}$, Elizabeth L.J. van Rensen ${ }^{4}$, Dorien L.M. Zwart ${ }^{5}$ \\ ${ }^{1}$ Department of Quality and Patient Safety, University Medical Center Utrecht, The Netherlands \\ ${ }^{2}$ Utrecht School of Governance, The Netherlands \\ ${ }^{3}$ Department of Woman and Baby, University Medical Center Utrecht, The Netherlands \\ ${ }^{4}$ Corporate Policy Unit of The Executive Board, University Medical Center Utrecht, The Netherlands \\ 5 Julius Research Center, University Medical Center Utrecht, The Netherlands
}

Received: February 4, 2018

DOI: $10.5430 /$ jha.v7n2p23
Accepted: March 11, $2018 \quad$ Online Published: March 19, 2018

URL: https://doi.org/10.5430/jha.v7n2p23

\begin{abstract}
Objective: Serious adverse events occur in healthcare, and do not solely have consequences for patients (first victims), but also affect physicians involved (second victims). These second victims experience diminished emotional well-being and less professional performance. An increasing number of hospitals organize support for second victims, although scientific evidence on the kind of support that is expected and needed is poor. This study therefore investigates support needs after serious adverse events from both personal (physicians) and organizational (quality and safety staff members) perspectives.

Methods: We conducted semi-structured interviews in a Dutch university medical center. Physicians $(\mathrm{N}=19)$ who had been directly involved in a serious adverse event participated. In addition, quality and safety staff members $(\mathrm{N}=3)$ reflected on the support needs as expressed by physicians. Verbatim transcripts were three-fold coded, which led to several themes for our inductive thematic analysis.

Results: Contrary to recent developments in healthcare organizations, participants did not plea for a hospital-wide support team. Acceptance of the emotional and professional impact of an adverse event by direct colleagues and supervisors is more important. Where such a cultural context is provided, physicians prefer support of a close and reliable colleague to cope with emotions and doubts, a supervisor who monitors recovery, and a healthcare organization that provides information about required procedures to learn from the events. However, this ideal was seldom found in the competitive working environment. This underlines the need for a shift from a competitive professional culture into a more supportive one.

Conclusions: Ideally, direct colleagues support each other after an adverse event. This form of collegial support can only be successful if there is general acceptance of healthcare professionals' vulnerability and their support needs within the direct working environment. To create the right circumstances to meet these support needs, both healthcare professionals and hospital organizations have to recognize and take their responsibilities.
\end{abstract}

Key Words: Second victims, Adverse events, Support needs, Organizational culture

\section{INTRODUCTION}

Saving lives under stressful circumstances evokes emotions of heroism. This sharply contrasts with a physician's feel- ings after a case in which a patient is accidentally harmed or killed, ${ }^{[1]}$ also known as an adverse event. ${ }^{[2]} \mathrm{Wu}$ described how such events not only have an impact on the patient (first

\footnotetext{
* Correspondence: Esther H.M. Leferink; Email: E.H.M.Leferink@umcutrecht.nl; Address: University Medical Center Utrecht, Heidelberglaan 100, Postbus 85500, 3508 GA Utrecht, The Netherlands.
} 
victim), but also on the professional involved (second victim). ${ }^{[3]}$ The second victim is thus defined as "a healthcare provider who is involved in an unanticipated adverse patient event, a medical error and/or a patient-related injury, and becomes victimized in the sense that the provider is traumatized by the event". ${ }^{[4]}$

Second victims feel confused and experience emotions of anger, anxiety, guilt, grief, self-criticism, and shame..$^{[5-15]}$ These emotions have an impact on both their professional and personal lives. ${ }^{[14,15]}$ Second victims therefore need time to recover from adverse events. Their recovery heavily depends on the extent to which second victims feel supported. ${ }^{[4,16-19]}$ Figures of burnout and depression, ${ }^{[20-22]}$ alcohol and drugs abuse, ${ }^{[23,24]}$ and even suicidal thoughts ${ }^{[25,26]}$ amongst second victims underline the importance of the issue. Despite these facts, healthcare workers experienced a lack of adequate support. ${ }^{[8,27,28]}$ Moreover, compared to other companies with high-risk professionals, hospitals' investment in support for second victims is lagging. ${ }^{[29]}$

This backlog may be related to the high standards set in the medical profession. Society, ${ }^{[30]}$ patients and family,,${ }^{[15]}$ and hospitals and colleagues ${ }^{[10]}$ have great difficulties dealing with second victims' vulnerability. ${ }^{[31]}$ Emotions related to events in which patients are accidentally harmed or killed are often not accepted. ${ }^{[32]}$ Second victims do not feel free to talk openly about their feelings, as this is seen as a sign of weakness. ${ }^{[4]}$ Successful implementation of support teams for second victims may only be possible if the prevailing culture shifts from individual blaming to group responsibility, and to mutual trust and openness amongst colleagues. ${ }^{[33-35]}$

Recent studies underline the importance and significance of organizational support for second victims' recovery. ${ }^{[36,37]}$ In line with these findings, an increasing number of hospitals have implemented a second victim support program over the last few years. In the United States, there are three leading hospitals. ${ }^{[38,39]}$ In Brigham and Women's Hospital, a Center for Professionalism and Peer Support was set up. ${ }^{[40-42]}$ In Johns Hopkins Hospital, a support program called RISE (Resilience in Stressful Events) has been implemented. ${ }^{[19,39,43]}$ The University of Missouri Health Care has its forYOU Team. ${ }^{[4,45]}$ All three support programs similarly provide a hospital-wide support team with well-trained peer supporters to help professionals involved in adverse events with their recovery. ${ }^{[39]}$ By doing so, these hospitals attempt to create a more open safety culture. ${ }^{[37,46]}$

So, adverse events cause second victims to feel emotionally and professionally exhausted within an environment that poses extremely high demands. Implementation of a hospital-wide support team and a change of culture should help them recover faster and better. Surprisingly, we do not exactly know what second victims regard as ideal form of support, ${ }^{[42]}$ nor how they expect this to be organized. ${ }^{[38]}$ The aim of our study is therefore to explore personal and organizational perspectives on support needs by interviewing physicians and quality and safety staff members.

\section{Methods}

\subsection{Study design and setting}

With a qualitative study approach, we examined interviewees' experiences and their expectations on types of support. We conducted one-to-one semi-structured interviews. This way of data collection was preferred, because it enabled us to start with open-ended questions derived from literature, and also gave interviewees an opportunity to add new themes, ${ }^{[47]}$ which was useful because only little scientific knowledge was available on our topic. Moreover, it enabled the researchers to create a safe and confidential setting in which the interviewee would feel comfortable to share his/her thoughts on personally delicate matters.

This study was conducted in a Dutch university medical center. During our data collection, this hospital provided no other forms of organized support than the support of a legally required team of well-trained social workers and nurses. An interdisciplinary research team of medical and social scientists worked together on this study. Suggestions for relevant literature data and access to interviewees were provided via members of the medical staff. A semi-structured guideline was designed, and interviews were held by a social scientist.

\subsection{Study sample}

We recruited physicians with various surgical and diagnostic professional backgrounds ( $\mathrm{N}=19$; 9 men, 10 women; 16 medical specialists consisting of 6 gynecologists, 6 anesthesiologists, 3 intensivists, and 1 psychiatrist, and 3 junior doctors in gynecology or geriatrics) who all had experienced a serious adverse event (resulting in severe harm or death) at least once during their career. In addition, three quality and safety staff members were interviewed to provide information about the organizational willingness to support employees. All interviewees were recruited through snowball sampling, ${ }^{[48,49]}$ starting from two key informants, i.e. two senior staff members of the hospital's gynecology department and the quality and safety service, respectively. Employees from both departments received an e-mail to volunteer in this research. Some physicians who learned about our study by chance also replied to our request. Interviews were conducted in January, February and March 2016, until we reached a saturation point at which no new insights came forward from the interviews. ${ }^{[50]}$ 


\subsection{Data collection}

Interviews took between 45-60 minutes and were situated at a location preferred by interviewees. Private settings in which they felt free to talk openly, such as their own workplace or at home, were chosen in order to encourage interviewees to share their emotional experiences of fallibility and vulnerability.

The semi-structured interviews started with an explanation of the aim of the research and an emphasis on the anonymity of interviewees. First questions of our guideline focused on interviewees' thoughts on the concepts "second victim" and "adverse event", to make sure that all interviewees were talking about the same topic. After these conceptual questions, interviewees were invited to talk openly on what impact adverse event(s) had on them. Based on Scott et al.'s "second victim phenomenon", the interviewer asked interviewees to take one impactful event in mind and to describe their recovery and experienced support. Interviewees were invited to discuss several aspects of processing the impactful event experienced. Interviews ended with the open question on interviewees' opinions on what helpful types of support for them in case of future events might be.

After analyzing the interviews with physicians, an interview guide was made to structure the interviews with the quality and safety staff members. These also started with questioning the concepts used, followed by presenting physicians' thoughts and views on impact, recovery and support. The quality and safety staff members were asked to reflect on physicians' experiences and expectations from an organizational point of view. This added insights to the ability to organize and structure peer support.

All interviewees agreed to audio recording the interviews. We transcribed the recordings verbatim. The interviewer also made notes during the interviews and summarized these at the end of each session to check if answers were understood properly. With this approach, some respondents were compelled to add something new or to explain their opinion in more detail.

\subsection{Data analysis}

Transcripts were coded in three different ways, ${ }^{[51]}$ for which we used NVivo qualitative data analysis software, version 11. The first step, open coding, gave us the opportunity to explore transcripts and to create tentative labels. Secondly, axial coding, was applied to derive topics and to relate codes. Thirdly, selective coding gave structure to data by relating codes to the main themes. The themes used for our inductive thematic analysis, were "second victim", "adverse event", "emotions", “coping strategies", "need for support", "expec- tations of support", and "organizational culture".

\subsection{Data reporting}

Results were reported as they emerged from data analysis. Quotes were used to illustrate the results and were translated from Dutch into English.

\section{RESUltS}

Starting the interviews by explaining the definition of the concepts "second victim" and "adverse event", interviewees (physicians) often responded that it felt wrong to typify them as victims. They stated that this term does not fit their image of being a professional, and that it is wrongly chosen, because it is primarily the patient who is the victim of an adverse event. They regarded the description "healthcare professional involved in an adverse event" as a more suitable label.

"You should never ask men whether they are scared, because they will always say 'no', even though they sometimes are. Physicians act in the same way. So, you should never ask a physician whether he is a victim, because he will always answer 'no', even though he sometimes is." (Physician D)

\subsection{Experienced emotions and coping}

Although interviewees (physicians) disliked being called "second victim", their descriptions of experienced emotions and coping used did meet the definition and associated characteristics as described in literature. All interviewees (physicians) had felt anger, failure, guilt, self-doubt, sadness, and disbelief, which underlines the impact of the unintended events. We could distinguish three ways in which coping with these emotions occurred.

First, there were some interviewees (physicians) who had spoken about the event with a colleague several times. This had relieved them emotionally. Informal collegial conversations and formal team discussions about the specific case and about practicing their work had helped restoring their professional confidence. What had supported them most, was the combined reflection on the event from both an emotional and the professional point of view. This group of interviewees admitted that the event had seriously hit them, but that they had been able to overcome strong emotions through support within their direct environment.

"There is a constant need for balance between criticism and support. A team meeting is the right moment to discuss these issues, because then others see that more people deal with these problems, that we can help each other, and that it is O.K. to talk about it." (Physician I)

Secondly, some interviewees (physicians) had not received 
much support, and had experienced a lack of attention for their feelings from their direct colleagues and supervisor. They had struggled to find someone with whom they could reflect on the events. For some of them, the only way to get support was visiting an external expert, such as a psychologist or a personal coach outside the hospital. This group had experienced deprivation of support and therefore blamed the hospital for not being a good employer in this sense.

"My hospital is far behind. My insurance company just said: 'You can call this phone number for help, because we do not want you to go on sick leave.' In my hospital, it is completely different. It struck me that my employer does not care about absenteeism that much. If the organization really cared, it would have put structural support for healthcare staff in place, but it did not." (Physician F)

Thirdly, a minority of the interviewees (physicians) argued that they had felt no need to talk with others. According to them, experiencing feelings are an integral part of their profession, and physicians need to be strong enough to overcome such events on their own. At the same time and quite to the contrary, these interviewees also admitted that it was not always the best way to cope with emotions. They stated that their ideas on what constitutes a real and strong professional was the result of having grown accustomed to working in a competitive, professional culture.

"As you can see, I am an older specialist. I studied in times where talking about one's emotions was uncommon, so I am used to solving my own problems. In general, I do not think that is a good way to cope with events, even though it is my primary response." (Physician $\mathrm{S}$ )

\subsection{Changing professional culture}

Most of the interviewees (physicians) described the professional culture within their direct working environment as competitive rather than supportive. Showing fallibility or vulnerability was often considered a weakness. Therefore, most interviewees were hesitant towards their colleagues and supervisor, both on admitting that an adverse event had taken place and on asking for help. Interviewees (physicians) underlined the profound influence of supervising professionals on the professional culture within medical teams.

"Did you feel safe to tell your supervisor about the event?' (Interviewer)

“No.” (Physician T)

"What if more physicians experience the same. What should your hospital do for them?" (Interviewer)

"There must be a confidential advisor, and we have such a person in this hospital. But to be honest, I think this is a non-discussion. If you do not feel safe to tell your supervisor what happened, then there is something very wrong in the organization.” (Physician T)

In changing the professional culture from a competitive into a more supportive one, interviewees (physicians) recommended their hospital to do two things. First, on a hospitalwide level, it could revise its selection criteria for supervising positions. Besides other job requirements (e.g. number of articles published, and level of medical performance), communicative skills such as being empathic and able to recognize support needs should be valued high in application procedures. Secondly, within medical teams, the hospital organization should stimulate intercollegiate communication about adverse events in order to normalize professionals' support needs. This should preferably be done by providing tools to discuss expectations of collegial support after an adverse event occurred.

\subsection{Expectations of support within the team environ- ment}

A majority of the interviewees (physicians) experienced or expected a reliable, non-judgmental and empathic colleague from within their direct working environment as the best supporter. In line with the results as described above, some interviewees (physicians) were fortunate to find support in that way. However, for most of them this was no reality yet. Besides presenting ideas on how to broaden the cultural acceptance around support needs, interviewees (physicians) also gave suggestions on how specific needs should be met. Ideally, supporters should help to cope with emotions and to overcome doubts about professional skills. Supervisors should monitor recovery and provide further professional help if needed. In addition to support within the direct working environment, the hospital organization should provide better information on the institutional procedures that are required to investigate and learn from an adverse event. Interviewees (physicians) stated that uncertainty about internal and external investigations resulted in experiencing additional emotional stress.

"Once I was called for an internal investigation. I thought: ' $O$ dear, what to do? What should I say, and what not? And what do they expect from me?' Those were the first things that came to mind, and it was not even my own patient." (Physician L)

\subsection{A hospital-wide support team}

A minority of the interviewees (physicians) claimed that support within the direct working environment might interfere with team relations. Therefore, they favored a greater distance between the professional involved and the supporter. 
For them, a hospital-wide support team was a better solution. However, they also argued that professionals might face more difficulties to contact a more anonymous hospitalwide support team than a direct colleague they knew well and trusted. Most interviewees (physicians) were skeptical about a hospital-wide solution, because for them this would only emphasize the presence of a cultural problem with the acceptance of vulnerability and support needs.

\subsection{Reflections from an organizational perspective}

Three quality and safety staff members with various leading hierarchical positions within the hospital reflected on the physicians' interview-findings. They were positively surprised to learn that some interviewees had received adequate support from direct colleagues. They recognized physicians' hesitance to ask for support from the currently available support team. The legally required hospital-wide support team consists of professionals from social sciences, such as psychologists and social workers. Physicians are not represented in this team. None of our interviewees (physicians) had contacted this team during recent years. Some interviewees (physicians) indicated that they were barely aware of the existence of this support team, whereas others did not think that members of this team would be able to understand the medical context from their point of view. According to the quality and safety staff members, these remarks stress physicians' inability to acknowledge their vulnerability and need for help. They also point at the organizational limits in meeting professionals' specific needs on a hospital-wide level. Both physicians and quality and safety staff members recognize that individual professionals, as well as the hospital organization have to take their responsibility in creating the right culture to stimulate acceptance among physicians of their vulnerability after a serious adverse event. In addition, the organization's support offer should be tailored more to specific physicians' needs in case of a serious adverse event.

\section{Discussion}

This study explored healthcare professionals' experiences and views on forms of support in a Dutch University Medical Center. During this research, several related aspects emerged, like emotions experienced, coping used and the dominant role of professional culture. Knowledge about the influence of these aspects is a prerequisite for the acceptance of collegial support.

\subsection{Recovery process}

Comparable with former studies, interviewees (physicians) experienced intense emotions after an adverse event. ${ }^{[5-15]}$ In addition, our study brought up a distinction of three different ways in which interviewees (physicians) coped during their recovery process. In a hospital without an appropriate collegial support program, the extent to which support is given depends on the willingness of a close colleague to do so. If no support is provided within the direct working environment, interviewees (physicians) received help from an external professional (psychologist or personal coach), or no support at all.

\subsection{Favorite form of support}

Understandably, interviewees (physicians) felt most supported in cases where they got help from a direct colleague, with whom they could discuss both the medical and emotional aspects of the event. An empathic, non-judgmental, and reliable peer from their own team was regarded as the best supporter. Interviewees' (physicians) opinions that support should come from a trustable and well-trained colleague are similar to the aim of peer support programs in the three healthcare organizations in the United States mentioned earlier. ${ }^{[39]}$ In addition, our research showed that support should be provided at three distinctive levels, namely on coping with emotions, on overcoming professional doubts, and on the accessibility of information on obligatory institutional procedures after an adverse event. In line with the first layer in Scott's Three-Tiered Interventional Model of Second Victim Support, ${ }^{[45]}$ this research found that interviewees (physicians) preferred forms of support organized in their direct working environment over a hospital-wide support team. The experience of quality and safety staff members also confirmed this finding. Furthermore, this study underlines the importance of embeddedness of support in the current organizational structure, which makes it more accessible and more acceptable for physicians to ask for and get support.

\subsection{Competitive culture}

Another notable finding was the confirmation of physicians' restraint in admitting emotional impact of adverse events and asking for support, as the still prevailing culture. A culture that is consistent with the idea that fallibility and vulnerability are weaknesses and do not fit the medical profession. Several studies already indicate the need for a cultural transformation, so that no longer individual blaming, but the system approach and a supportive attitude should be prevailing. ${ }^{[33-35]}$ Our research shows that interviewees (physicians) still felt personally affected after an adverse event and that shared responsibility is not (yet) an integrated part of coping with the emotional aftermath.

\subsection{Towards cultural acceptance}

Overviewing these findings, our study stresses that providing the necessary support mainly requires a cultural shift towards acceptance of the need for support. Yet, our findings also 
indicate that healthcare professionals who experienced an adverse event have difficulties to regard themselves as victims, as it would emphasize their fallibility and vulnerability. This seems to raise the bar for seeking support and for accepting support from others, because they are accustomed to working in a tough, competitive professional culture. Our interviewees (physicians) find the label "second victim" an unacceptable one, because it does no justice to the patient nor to themselves. A physician is expected to take responsibility after an adverse event and the term "victim" is incompatible with this view. Together with colleagues, $\mathrm{Wu}$, who introduced this term, ${ }^{[3]}$ recently initiated a debate about the appropriateness of the label "second victim" that revealed objections to the terminology similar to our empirical findings. ${ }^{[52]}$ Notwithstanding the terminology discussion, the need for support is undisputed. In promoting acceptance of vulnerability and in taking care of employees, executive boards and supervisors have an influential role within the hospital. They should recognize the need for support and encourage it in order to create favorable circumstances for the acceptance of support needs. Indeed, without a broad cultural acceptance, hurdles in getting support will remain.

\subsection{Limitations}

Our research has some limitations. All data were collected in one university hospital in the Netherlands, and the nineteen healthcare professionals that were interviewed were not randomly selected volunteers. Despite the relatively small number of interviewees (physicians and quality and safety staff members) we reached a point of saturation of the data variety. We think that our study reveals relevant and transferable findings for other institutions as our data stem from various clinical specialisms, all with their own distinct professional cultures. However, because of the voluntary-based participation, there might be a bias in our sample, as we mainly interviewed healthcare professionals who managed to cope positively with their emotional vulnerability. We will have missed professionals that are (still) not able to speak about the event and the impact it had on them. Nonetheless, the demographic characteristics and the coping strategies and recovery processes of our interviewees (physicians) show diversity rather than uniformity. This enhances the validity of our findings. For future research, we suggest involving not only physicians, but also other healthcare professionals, as they may equally be traumatized by an adverse event and might need support.

\section{Conclusions}

This study examined physicians' and quality and safety staff members' views on support needs after a serious adverse event. Physicians involved did not recognize themselves as being "second victims", because this term does not reflect their ideas about professionalism. It seems that victimization does not encourage the acceptance of vulnerability and support needs. In addition, this study indicates that receiving support is hard in a professional culture that is perceived as competitive rather than supportive. However, cultural acceptance of support needs is very important to allow physicians to be supported. Ideally, physicians should receive support from a direct peer who helps them cope with their emotions and reflect on their professional performance. In addition, the supervisor should monitor the recovery process, and hospitals should provide clear information about the required institutional procedures after an adverse event. In turning this ideal into reality, both healthcare professionals and hospital(s) have to recognize and take up their responsibility, first and foremost by showing exemplary empathic behavior and vulnerability and by stimulating the dialogue thereon.

\section{CONFLICTS OF INTEREST Disclosure}

The authors declare they have no conflicts of interest.

\section{REFERENCES}

[1] Newland J. Medical errors snare more than one victim. The Nurse Practitioner. Sep 2011; 36(9): 5. https ://doi .org/10.1097/01 .NPR.0000403294.04964.57

[2] Wagner C, Van der Wal G. Voor een goed begrip. Med. Cont. Nov 2005; 60(47): 1888-1891.

[3] Wu AW. Medical error: the second victim. The doctor who makes the mistake needs help too. BMJ Qual. Saf. Health Care. Mar 2000; 320(7237): 726-727.

[4] Scott SD, Hirschinger LE, Cox KR, et al. The natural history of recovery for healthcare provider 'second victim' after adverse patient events. BMJ Qual. Saf. Health Care. Oct 2009; 18(5): 325-330. PMid: 19812092. https://doi.org/10.1136/qshc. 2009.032870
[5] Han K, Bohnen JD, Peponis T, et al. The Surgeon as the Second Victim? Results of the Boston Intraoperative Adverse Events Surgeons' Attitude (BISA) Study. J. Am. Coll. Surg. Jan 2017; Pii: S1072-7515(17)30035-2.

[6] Abd Elwahab S, Doherty E. What about doctors? The impact of medical errors. Surgeon. 2014; 12: 297-300. PMid: 25056239. https://doi.org/10.1016/j.surge.2014.06.004

[7] O'Breine M, Sterling P, Palacios-Deflingher L, et al. Emotional impact of patient safety incidents on family physicians and their office staff. J. Am. Board Fam. Med. Mar-Apr 2012; 25(2): 177-83. PMid: 22403198. https ://doi.org/10.3122/jabfm.2012.02. 110166

[8] Gallagher TH, Waterman AD, Ebers AG, et al. Patients' and Physi- 
cians' Attitudes Regarding the Disclosure of Medical Errors. JAMA. Feb 2003; 289(8): 1001-1007. PMid: 12597752. https://doi.or $\mathrm{g} / 10.1001 / \mathrm{jama} .289 .8 .1001$

[9] Newman MC. The emotional impact of mistakes on family physicians. Arch. Fam. Med. Feb 1996; 5: 71-75. PMid: 8601210. https://doi.org/10.1001/archfami.5.2.71

[10] Christensen JF, Levinson W, Dunn PM. The heart of darkness: the impact of perceived mistakes on physicians. J. Gen. Intern Med. JulAug 1992; 7(4): 424-431. PMid: 1506949. https://doi.org/10 $.1007 / \mathrm{BF} 02599161$

[11] Wu AW, Folkman S, McPhee SJ, et al. Do house officers learn from their mistakes? JAMA. Apr 1991; 265(16): 2089-2094. PMid: 2013929. https://doi.org/10.1001/jama.1991.0346 0160067031

[12] Levinson W, Dunn PM. A piece of my mind. Coping with fallibility. JAMA. Apr 1989; 261: 2252. PMid: 2926972. https: //doi.org/10.1001/jama.1989.03420150102047

[13] Hilfiker D. Facing Our Mistakes. N. Engl. J. Med. Jan 1984; 310 : 118-122. PMid: 6690918. https://doi.org/10.1056/NEJM19 8401123100211

[14] Schelbred AB, Nord R. Nurses' experiences of drug administration errors. J. Adv. Nurs. Nov 2007; 60(3): 317-324. PMid: 17908127. https://doi.org/10.1111/j.1365-2648.2007.04437.x

[15] Aasland OG, Førde R. Impact of feeling responsible for adverse events on doctors' personal and professional lives: The importance of being open to criticism from colleagues. BMJ Qual. Saf. Health Care. Feb 2005; 14(1): 13-17. PMid: 15691998. https: //doi.org/10.1136/qshc.2002.003657

[16] Chan ST, Khong PC, Wang W. Psychological responses, coping and supporting needs of healthcare professionals as second victims. Int. Nurs. Rev. Sep 2016. PMid: 27679402.

[17] Nelson WA, Beyea SC. The role of an ethical culture for the prevention and recovery of 'second victims'. BMJ Qual. Saf. Health Care. Oct 2009; 18(5): 323-324. PMid: 19812091. https://doi.org/ 10.1136/qshc. 2009.034843

[18] Seys D, Wu AW, Van Gerven E, et al. Healthcare professionals as second victims after adverse events: a systematic review. Eval. Health Prof. Jun 2013; 36(2): 135-162. PMid: 22976126. https://doi.org/10.1177/0163278712458918

[19] Edrees HH, Paine LA, Feroli ER, et al. Healthcare workers as second victims of medical errors. Pol. Arch. Med. Wewn. Apr 2011; 121(4): 101-108. PMid: 21532531.

[20] Shanafelt TD, Balch CM, Bechamps G, et al. Burnout and medical errors among American surgeons. Ann. Surg. Jun 2010; 251(6): 9951000. PMid: 19934755. https://doi.org/10.1097/SLA.0b01 3e3181bfdab3

[21] Schwappach DL, Boluarte TA. The emotional impact of medical error involvement on physicians: a call for leadership and organizational accountability. Swiss Med. Wkly. Jan 2009; 139(1-2): 9-15. PMid: 18951201.

[22] West CP, Huschka MM, Novotny PJ, et al. Association of perceived medical errors with resident distress and empathy: a prospective longitudinal study. JAMA. Sep 2006; 296(9): 1071-1078. PMid: 16954486. https://doi.org/10.1001/jama.296.9.1071

[23] Oreskovich MR, Kaups KL, Balch CM, et al. Prevalence of alcohol use disorders among American surgeons. Arch. Surg. Feb 2012; 147(2): 168-174. PMid: 22351913. https://doi.org/10.1001/ archsurg. 2011.1481

[24] Hughes PH, Conard SE, Baldwin DC jr, et al. Resident physician substance use in the United States. JAMA. Apr 1991; 265(16): 20692073. PMid: 2013925. https://doi.org/10.1001/jama. 1991. 03460160047027

Published by Sciedu Press
[25] Shanafelt TD, Balch CM, Dyrbye L, et al. Special report: suicidal ideation among American surgeons. Arch. Surg. Jan 2011; 146(1): 54-62. PMid: 21242446. https ://doi .org/10.1001/archsurg .2010 .292

[26] Center C, Davis M, Detre T, et al. Confronting depression and suicide in physicians: a consensus statement. JAMA. Jun 2003; 289(23): 3161-3166. PMid: 12813122. https://doi.org/10.1001/jama .289 .23 .3161

[27] Ullström S, Sachs MA, Hansson J, et al. Suffering in silence: a qualitative study of second victims of adverse events. BMJ Qual. Saf. Health Care. Apr 2014; 23(4): 324-331. https ://doi .org/10.1 136/bmjqs-2013-002035

[28] Waterman AD, Garbutt J, Hazel E, et al. The emotional impact of medical errors on practicing physicians in the United States and Canada. Joint Commission Journal on Quality \& Patient Safety. 2007; 33(8): 467-476. https://doi.org/10.1016/S1553-725 0 (07) 33050-X

[29] Santamauro CM, Kalkman CJ, Dekker SWA. Second victims, organizational resilience and the role of hospital administration. Journal of Hospital Administration. Apr 2014; 3(5): 95-103. https : //doi.org/10.5430/jha.v3n5p95

[30] DeVille K. God, science and history: The cultural origins of medical error. Sharpe VA ed. Accountability: Patient safety and policy reform. Washington, DC: Georgetown University Press; 2004. 143-158 p.

[31] Leape LL. Error in medicine. JAMA. Dec 1994; 272(23):1851-1857. PMid: 7503827. https://doi.org/10.1001/jama.1994. 0352 0230061039

[32] Vincent C. Patient Safety. London: Churchill Livingstone; 2006.

[33] Denham CR. TRUST: The 5 Rights of the Second Victim. J. Patient Saf. Jun 2007; 3(2): 107-119. https://doi.org/10.1097/01.j ps.0000236917.02321.fd

[34] Weick KE, Sutcliffe KM. Managing the unexpected: Resilient performance in an age of uncertainty. 2nd ed. San Francisco: Jossey-Bass; 2007.

[35] Reason J. Managing the Risks of Organizational Accidents. Hants (UK): Ashgate Publishing Ltd; 1997.

[36] Burlison J, Quillivan R, Scott S, et al. The effects of the second victim phenomenon on work-related outcomes: connecting self-reported caregiver distress to turnover intentions and absenteeism. Journal of Patient Saf. Nov 2016. https ://doi .org/10.1097/PTS. 000000 0000000301

[37] Harrison R, Lawton R, Perlo J, et al. Emotion and coping in the aftermath of medical error: a cross country exploration. Journal of Patient Saf. 2015; 11(1): 28-35. PMid: 25695552. https: //doi.org/10.1097/PTS.0b013e3182979b6f

[38] Edrees H, Federico F. Supporting clinicians after medical error: the needs of these 'second victims' are often ignored. BMJ. Apr 2015; 350: 1982. PMid: 25877670. https://doi.org/10.1136/bmj. h1982

[39] De Wit ME, Marks CM, Natterman JP, et al. Supporting second victims of patient safety events: shouldn't these communications be covered by legal privilege? Journal of Law Medicine \& Ethics. 2013; 41(4): 852-858. PMid: 24446943. https://doi.org/10.1111/ jlme. 12095

[40] Van Pelt F. Peer support: healthcare professionals supporting each other after adverse medical events. BMJ Qual. Saf. Health Care. Aug 2008; 17(4): 249-252. PMid: 18678720. https://doi.org/10.1 136/qshc. 2007.025536

[41] Shapiro J, Whittemore A, Lawrence CT. Instituting a Culture of Professionalism: The Establishment of a Center for Professionalism and Peer support. Jt. Comm. J. Quality Patient Safety. Apr 
2014; 40(4): 168-177. https://doi.org/10.1016/S1553-725 0 (14) 40022-9

[42] Hu YY, Fix ML, Hevelone MD, et al. Physicians' needs in coping with emotional stressors: the case for peer support. Arch. Surg. Mar 2012; 147(3): 212-217. PMid: 22106247. https://doi.org/10 .1001 /archsurg. 2011.312

[43] Edrees H, Connors C, Paine L, et al. Implementing the RISE second victim support programme at the Johns Hopkins Hospital: a case study. BMJ Open. 2016; 6(9): 1-12. PMid: 27694486. https://doi.org/10.1136/bmjopen-2016-011708

[44] Merandi J, Liao N, Lewe D, et al. Deployment of a second victim peer support program: a replication study. Pediatric Qual. \& Saf. June 2017; 2(4): 1-8.

[45] Scott S, Hirschinger L, McCoig M, et al. Caring for our own: deploying a systemwide second victim rapid response team. Jt. Comm. J. Qual. Patient Saf. May 2010; 36(5): 233-240. https: //doi.org/10.1016/S1553-7250(10) 36038-7

[46] Putnam K. Peer support programs help health care workers overcome emotional trauma. Periop Briefing. 2016; 104(3): 7-9.
[47] Baarda DB, De Goede MPM, Teunissen J. Basisboek Kwalitatief Onderzoek. Groningen: Stenfert Kroese; 2005.

[48] Atkinson R, Flint J. Accessing hidden and hard-to-reach populations: snowball research strategies. Social Research Update. 2001; 33(1): $1-4$.

[49] Reed MS, Graves A, Dandy N, et al. Who's in and why? A typology of stakeholder analysis methods for natural resource management. Journal of Environmental Management. 2009; 90: 19331949. PMid: 19231064. https://doi.org/10.1016/j.jenvma n.2009.01.001

[50] Guest G, Bunce A, Johnson L. How many interviews are enough? An experiment with data saturation and variability. Field Methods. 2006; 18(1): 59-82. https://doi .org/10.1177/1525822X05279903

[51] Boeije HR. Analysis in qualitative research. London: SAGE Publications Ltd; 2010.

[52] Wu AW, Shapiro J, Harrison R, et al. The impact of adverse events on clinicians: what's in a name? J. Patient Saf. 2017. https: //doi.org/10.1097/PTS.0000000000000256 